\title{
MAC Protocol Operation in Energy Harvesting for 5G Networks
}

\author{
Joydev Ghosh*, Zoltán Jakó ${ }^{2}$ \\ ${ }_{2}^{1}$ National Research Tomsk Polytechnic University, Russia, \\ Budapest University of Technology and Economics, Hungary.
}

\begin{abstract}
Two vital challenges arise in the next generation 5G networks: scarcity of spectrum and the aspect of energy efficiency (EE). In this letter, we first formulate the prime issue, i.e., energy harvesting (EV) for $\mathbf{5 G}$ wireless networks, in which spectrum and energy resources are efficiently received and resourcefully managed. Thereafter, we introduce a medium access control (MAC) scheme for dual tier 5G networks; it also invokes the simultaneous wireless information and power transfer (SWIPT) technique with other data transmission schemes, i.e., cooperative transmission (CT) scheme and non-cooperative transmission (NCT) scheme with EV abilities. Illustrative results demonstrate significant improvement in aggregated throughput and EE.
\end{abstract}

Keywords: SWIPT technique, MAC Protocol, Energy Harvesting, NCT scheme, 5G

Received on 22 January 2017, accepted on 7 August 2017, published on 10 January 2018

Copyright (C) 2018 Joydev Ghosh, Zoltán Jakó, licensed to EAI. This is an open access article distributed under the terms of the Creative Commons Attribution licence (http://creativecommons.org/licenses/by/3.0/), which permits unlimited use, distribution and reproduction in any medium so long as the original work is properly cited.

doi: 10.4108/eai.10-1-2018.153554

\section{Introduction}

An interesting metrics in next generation wireless networks is energy supply in a cooperative cognitive radio network (CCRN) and this can be one of the most crucial issues to reduce the cost of an entire network. Therefore, a emerging technique, energy harvesting (EV), is introduced to elongate lifetime of wireless network in a much safe and better way, i.e., harvesting energy from ambient environment [1], [2] and radio frequency (RF) signal. The application of EV to CCRN has been reviewed in [3]. In simultaneous wireless information and power transfer (SWIPT) technique, a user at the receiving end utilizes the same received signal to decode information and also for harvesting energy. Besides, the SWIPT technique is divided in two modes, Time Switching (TS) mode and Power Splitting (PS) mode [4].

*Corresponding author. Email:joydev.ghosh.ece@gmail.com
In this letter, we introduce a CCRN with EV and analyze the performance of MAC protocol operation for $5 \mathrm{G}$ networks. The Nakagami- $m$ fading channel has been included in the simulation testbed as it provides better approximated results in contrast to the Rayleigh fading channel [5]. The major contributions of this letter can be summarized as follows:

- We analyze theoretically Harvested Energy at the secondary transmitter (ST) in a cooperative transmission (CT) scheme over Nakagami- $m$ fading channel. The EV with femtocells is a new challenging topic.

- A MAC protocol scheme has been introduced with heterogenous spectrum sensing and EV capabilities. Illustrative results on the proposed protocol provide useful insights in energy efficiency (EE) and spectral efficiency (SE) for $5 \mathrm{G}$ networks.

- We compare the performance of the proposed MAC protocol scheme, CT scheme and non- 
cooperative transmission (NCT) scheme. Most importantly, we probe the trade-off between network performance of energy consumption and aggregated throughput in details.

\begin{tabular}{|l|l|l|}
\hline $\mathrm{uV}:$ ST to PR & $\mathrm{u}(1-v):$ ST to SR & $(1-u):$ PT to ST \\
\hline
\end{tabular}

Fig. 2: Segmentation of phase

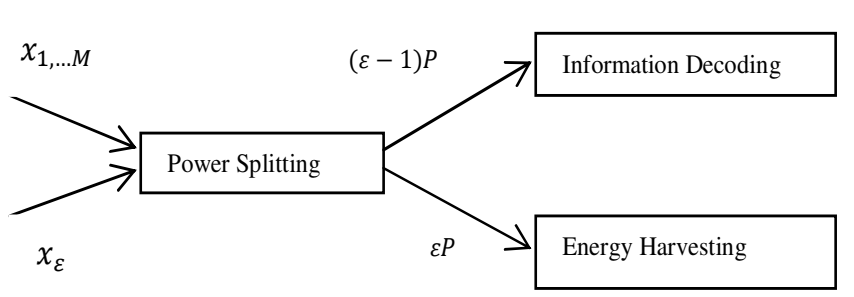

Fig. 3: Energy Harvesting Protocol
Fig. 1: System Model

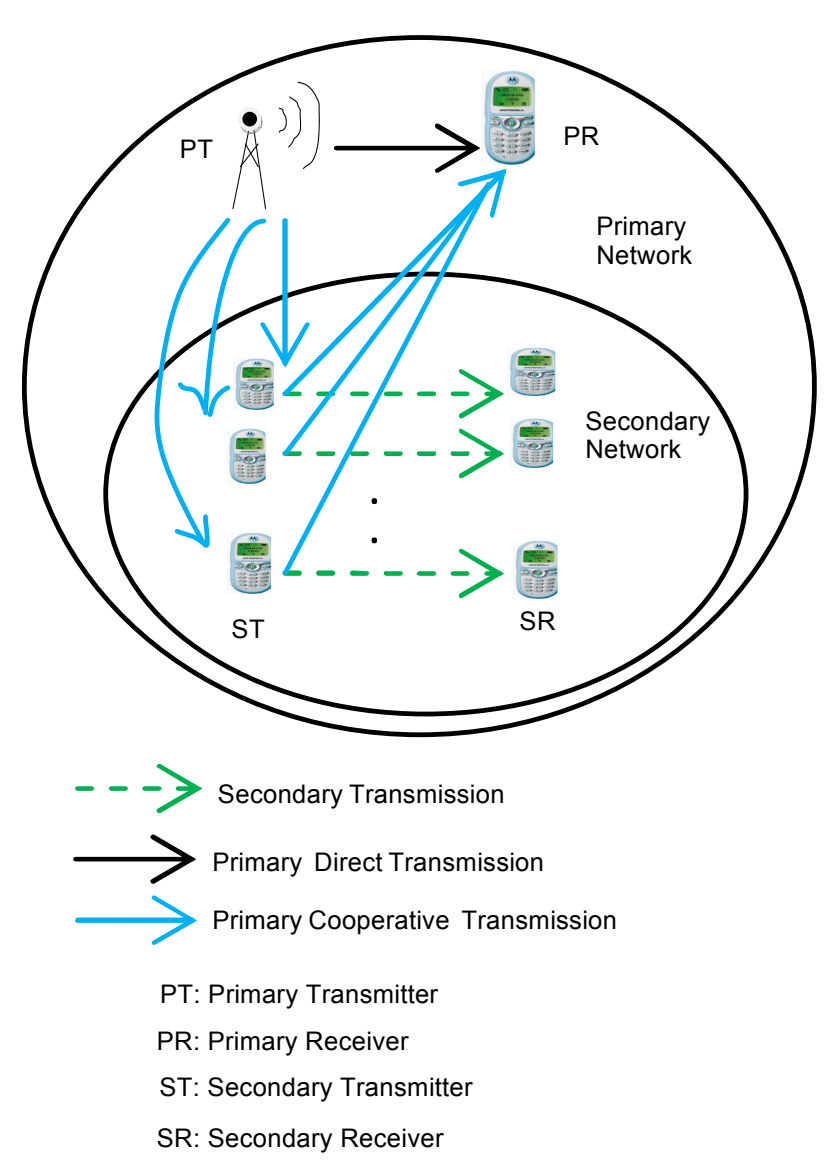

\section{System Model}

According to Fig.1, we introduce a CCRN where a macrocell network coexists with a femtocell network. The Macrocell network consists of single macro user (MU) and it further composed of a transmitter and a receiver, while in comparison, the femtocell network includes $F$ denotes femto users (FUs), i.e., $F$ denoted the number of transmitter and receiver units. Furthermore, we assume that MU can share their licensed band with secondary networks for exchange of cooperation. Hence, FUs can get benefits, meanwhile, MU's need of quality of experience (QoE) can be fulfilled. Besides, independently and identically distributed (i.i.d) Nakagami-m fast fading is assumed for all channels in the model.

\section{A. Phase Splitting and EV}

Let variable $\varphi(|\varphi|=F)$ and $\emptyset(|\varnothing|=x \leq F)$ indicate the collections of objects, i.e., entire STs in the femtocell network and the set of entire STs have the opportunity to involve in coordination, respectively. Without any violation of generality, we consider the time duration for transmission is unity, and split up the entire phase into a number of segments as depicted in Fig. 2. The $1^{\text {st }}$ phase denoted by $u v(0<u<1)$ is the time interval to perform EV by the STs. In the $2^{\text {nd }}$ phase, denoted by $u(1-v)(0<v<1)$, entire STs considered in the $1^{\text {st }}$ phase forward information to SR. Finally, in the $3^{\text {rd }}$ phase, denoted by $(1-u)$, every considered PT forwards its own information to the respective receiver.

In addition, the PS mode has been applied, where every ST can divide the entire power of the received signal into two components as depicted in Fig. 3: the first one, i.e., $\varepsilon P$, for $\mathrm{EV}$ and the second one, i.e., $(1-\varepsilon) P$ for information decoding of the received signal in connection to the PT, where $\varepsilon$ denotes the fraction of received signal power $(P)$. Nevertheless, STs also harvest energy from interferers and RF signals $\left(I_{n}, n \in[1, M]\right)$ [6].

\section{B. Cooperative Transmission (CT) Scheme}

In the first phase, the signal received by the $x^{\text {th }}$ $(0<x<F)$ ST can be expressed as

$$
S_{1 x}=\sqrt{P_{P T}} h_{P x S} I_{P T}+\sum_{n=1}^{M} \sqrt{P_{n x}} g_{n x} I_{n}+\zeta_{1 x}
$$


where $P_{P T}$ indicates the transmit power (TP) of PT, $h_{P S x}$ indicates the channel gain of PT to $x^{\text {th }}, I_{P T}$ indicates the transmit signal from PT, $M$ indicates the number of interferers at ST, $P_{n x}$ indicates TP from the $n^{\text {th }}$ interferer to the $x^{\text {th }} \mathrm{ST}, g_{n x}$ indicates the channel gain from the $n^{\text {th }}$ interferer to the $x^{\text {th }} \mathrm{ST}, I_{n}$ indicates transmitting signal of the $n^{\text {th }}$ interferer, $\zeta_{1 x}$ indicates i.i.d Additive White Gaussian Noise (AWGN) with zero mean and variance equals to $N_{0}$ at the $x^{\text {th }} \mathrm{ST}$.

In the second phase, the signal received by the PR associated to the $x^{\text {th }}$ ST can be expressed by

$$
S_{2 x}=\sqrt{P_{S T}} h_{S x P} I_{x S T}+\zeta_{2 x}
$$

where $P_{S T}$ indicates TP of ST, $h_{S x P}$ indicates the channel gain of the $x^{\text {th }} \mathrm{ST}$ to PT, $I_{x S T}$ indicates the transmit signal from the $x^{\text {th }} \mathrm{ST}, \zeta_{2 x}$ indicates i.i.d AWGN with zero mean and variance equals to $N_{0}$ at PR associated to the $x^{\text {th }} \mathrm{ST}$.

Furthermore, we consider that every ST in the set of $\varphi$ has the capability to decode information successfully, i.e., both $I_{P T}$ and $I_{x S T}$ are treated as the same. In the first phase, the PS mode has been applied by ST with $\varepsilon$ $(0<\varepsilon<1)$, the observation on the received signal in connection with the $x^{\text {th }} \mathrm{ST}$ to EV can be expressed as

$S_{x}=\sqrt{\varepsilon P_{P T}} h_{P x S} I_{P T}+\sqrt{\varepsilon} \sum_{n=1}^{M} \sqrt{P_{n x}} g_{n x} I_{n}+\sqrt{\varepsilon} \zeta_{1 x}$

Hence, the harvested energy can be expressed by [11]

$E_{x}=u v \varepsilon \eta\left(P_{P T}\left|h_{P x S}\right|^{2}+\sum_{n=1}^{M} \sqrt{P_{n x}}\left|g_{n x}\right|^{2}\right)$

where $\eta$ indicates an energy conversion factor while STs store harvested energy into their storage devices, $u v$ indicates that the EV takes place only in the first phase, $\sum_{n=1}^{M} \sqrt{P_{n x}}\left|g_{n x}\right|^{2}$ introduces from the end of interferers at STs. Therefore, it is quite obvious that the harvested energy is proportional to $u, v, \varepsilon, \eta$.

It is also assumed that harvested energy in STs is adequate for the transmission of ST to PR. Thereafter, harvested energy of STs belong to the set of $\varphi$ are not less than $Q_{0}$ (i.e., $E_{x} \geq Q_{0}$ ) would have selected from $\varphi$ and placed into $\emptyset(|\varnothing|=x \leq F)$ as a ST having opportunity for coordination [10].

\section{Our Proposed MAC Protocol}

A sensor network is comprised of a large number of limited power sensor nodes which collect and process data from a target domain and transmit information back to specific sites (e.g., headquarters, disaster control centres) [8]. We consider wireless sensor networks which share the same wireless communication channel. A
Medium Access Control (MAC) protocol specifies how nodes share the channel, and hence plays a central role in the performance of a sensor network. Sensor networks contain many nodes, typically dispersed at high, possibly non-uniform, densities; sensors may turn on and off in order to conserve energy; and, the communication traffic is space and time correlated. Contention occurs when two nearby sensor nodes both attempt access the communication channel. Contention causes message collisions, which are very likely to occur when traffic is frequent and correlated, and they decrease the lifetime of a sensor network [9]. A MAC protocol is contention-free if it does not allow any collisions. A contention-free MAC protocol should be able to bring the network from an arbitrary state to a collision-free stable state. Since the protocol is distributed, during this stabilization phase collisions are unavoidable. In the stable state, we measure the efficiency by a node's throughput, the inverse of the time interval between which it is allowed to transmit messages by the MAC protocol.

We introduce MAC protocol operation based on cooperative sensing (CS) for an EV-CR network, where the FUs can apply CS to improve their sensing performance. In the CS mode, the FUs deployed with an EV module will sense the licensed channels (LCs) to find the unoccupied channel (i.e., the channel that is not used by MU), or to check whether their unconsumed energy goes beyond a preset threshold or not. However, we assume EV heterogeneity, which can be stated as the different capabilities to collect energy from the ambient environment for different FUs. Besides, different FUs may have separate sensing capabilities. The different

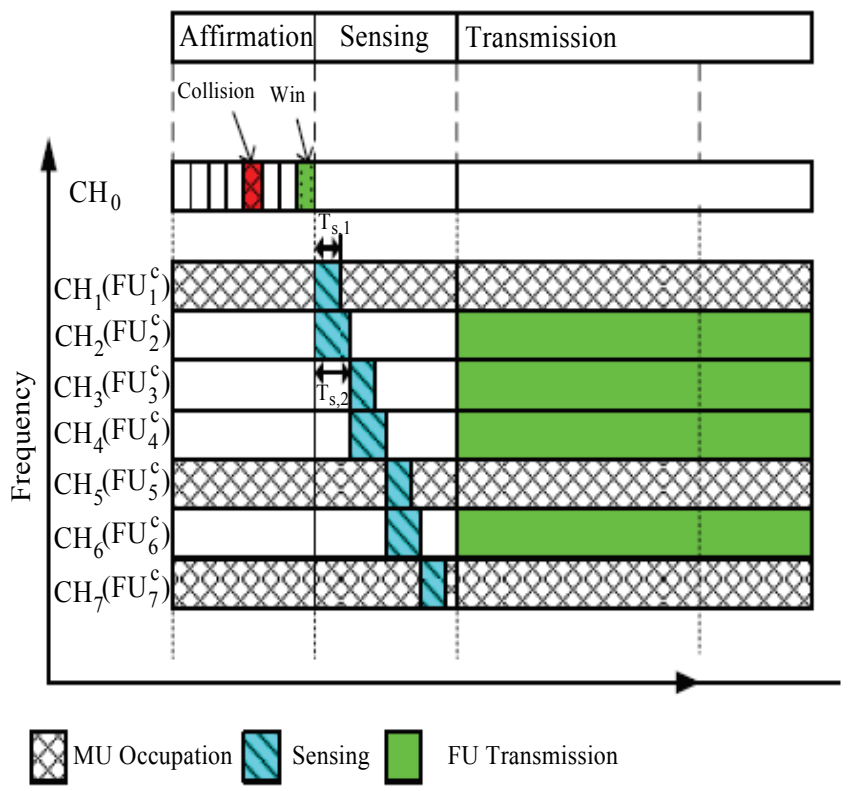

Fig. 4: Different Phases of MAC Protocol Operation 
phases of the MAC protocol operation are depited in Fig. 4. The entire phase in MAC protocol operation is split up into three stages: affirmation stage, sensing stage and transmission stage.

At the affirmation stage, when FUs have data to forward, it initially follows the control channel. If there is nothing found regarding the exchange of information, the FU sends a signal to the predetermined receiver to book the control channel. In the sensing stage, the FU transmits sensing signal regarding the essentiality of the CS mechanism and halts for other FUs to join in this sensing process by sending a reply signal with the information on the status of their energy. When an automatic mechanism for activating a device at a preset time is expired, the FU evaluates the optimum number of the cooperative all FUs and the amount of residual energy used by each FU in sensing. Thereafter, entire FUs begin CS mechanism. Once all channels are sensed, the FU shares information with all cooperative FUs for unoccupied channels. In the transmission stage, cooperative data transmissions of the FUs happen simultaneously over the available channels.

\section{Throughput Performance}

A Remarkable amount of energy is consumed by the cooperative FUs that may cause the energy scarcity at the sensing stage and transmission stage. Thus, cooperative FUs and the consumption of energy in CS are two important design variables to balance this initial tradeoff between the obtainable throughput $y(t)$ and the sensing overhead $z(t)$.

The difference between the throughput and the sensing overhead defines the aggregate throughput of the femtocell based 5G networks. Let $\psi$ indicates the whole amount of licensed channels. Let $\Theta_{s, x}(t)$ represents the consumption of energy for CS by the $x^{\text {th }}$ FU during the time slot $t$. We formulate an optimization problem to balance the achieved throughput and the sensing overhead as follows

$$
\begin{gathered}
\max _{\substack{F, \varphi_{s, i} \\
x=1,2, \ldots, F}} \lim _{T \rightarrow \infty} \frac{1}{T} \sum_{t=1}^{T}[y(t)-z(t)] \\
\text { s.t.r } \Theta_{s, x}(t) \leq E_{r, x}(t)-E_{o, x}, \\
E_{r, x}(t+1)=\min \left\{E_{r, x}(t)+E_{x}(t)-r \Theta_{s, x}(t), E_{c}\right\} \\
x=1,2, \ldots, v ; \quad t=1,2, \ldots, T
\end{gathered}
$$

where $r=\frac{\psi}{v}$ indicates the number of CS round, $E_{r, x}(t)$ indicates the unconsumed energy at the $x^{\text {th }} \mathrm{FU}$ during the time slot $t, E_{o, x}$ indicates the energy threshold for the $x^{\text {th }}$ $\mathrm{FU}, E_{c}$ indicates the capacity of the storage device for all FUs, $\left\{E_{x}(t) x=1,2, \ldots, v\right\}$ indicates the harvested energy by each FU during time slot $t$ and it is prototyped of an i.i.d sequence of random variables with mean value $\mathbb{E}\left[E_{x}(t)\right]=\overline{E_{x}}$.

\section{Results and discussion}

In this section, we assess the performance of introduced MAC protocol operation with the CT scheme and the NCT scheme. In the NCT scheme, each FU has to observe a set of LCs subsequently by itself. In CT scheme, each FU arbitrarily selects one of the LCs for sensing without taking the cautious option of the cooperative FUs as well as the consumption of energy during sensing. In the simulation testbed, 30 FUs and 10 LCs are included. The channel bandwidth (BW) is $1 \mathrm{MHz}$. The time duration of a frame is 1second. The sensing power (SP) and TP of a FU are $110 \mathrm{~mW}$ and $210 \mathrm{~mW}$, respectively. Additionally, $E_{o, x}=0.1 \mathrm{~J}$ and $E_{c}=1 \mathrm{~J}$.

Fig. 5 illustrates the comparison of the consumption of energy among the MAC protocol scheme, CT scheme and NCT scheme. It can be noticed that the introduced MAC protocol scheme is used to spend a smaller amount of energy compared to the CT scheme. The reason behind this phenomenon is that the MAC protocol only permits the optimum number of FUs to join with the CS scheme. In the intervening period of time, the sensing energy used up by each cooperative FU is conscientiously decided. Thus, the consumption of energy at the sensing stage can be significantly minimized. Furthermore, the proposed protocol consumes more energy in contrast to the NCT scheme. Therefore, in the MAC protocol, the CS requires sensing aid from other FUs which causes additional consumption of energy. Although, this energy consumption can achieve elevated throughput as depited in Fig. 6.

Fig.6 illustrates the aggregate throughput of the MAC protocol scheme, in contrast to the CT scheme and NCT scheme. The difference indicates that the introduced MAC protocol scheme is capable to obtain much larger throughput than the CT scheme and NCT scheme. The phenomenon can be described as follows. At the sensing stage, the MAC protocol is capable to probe and detect more unoccupied channels, than that of the NCT scheme. The CT scheme uses entire FUs to join in the CS mechanism which enhances the sensing overhead and degrades the throughput. On the other hand, the proposed MAC protocol initiates the optimum number of the cooperative FUs to guarantee against the condition that the net cooperative sensing aloft to be restricted, this further leads to even more throughput. As a result, the MAC protocol scheme is capable of obtaining elevated throughput with lower-level sensing overhead. As illustrated in the simulation outcomes, the suggested MAC protocol scheme can obtain even better network operation in contrast to other two schemes, particularly for $5 \mathrm{G}$ networks. 


\section{Conclusion}

In this letter, we proposed a MAC protocol operation based framework to improve the overall performance of $5 \mathrm{G}$ networks. Initially, we presented the PS and EV model, CT model, and MAC protocol model to get useful insights in harvested energy. Thereafter, an EV based MAC protocol operation, compatible in a CR aided 5G wireless networks, has been demonstrated to show appreciable improvement in aggregated throughput and EE.

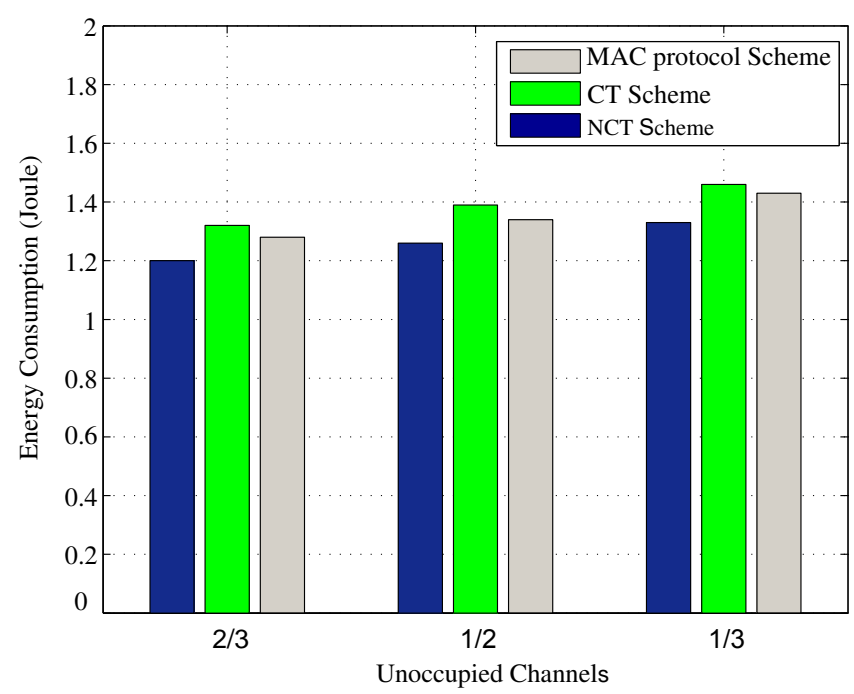

Fig. 5: Comparison of Different Schemes in Terms of Energy Consumption for $\varepsilon=0.6$

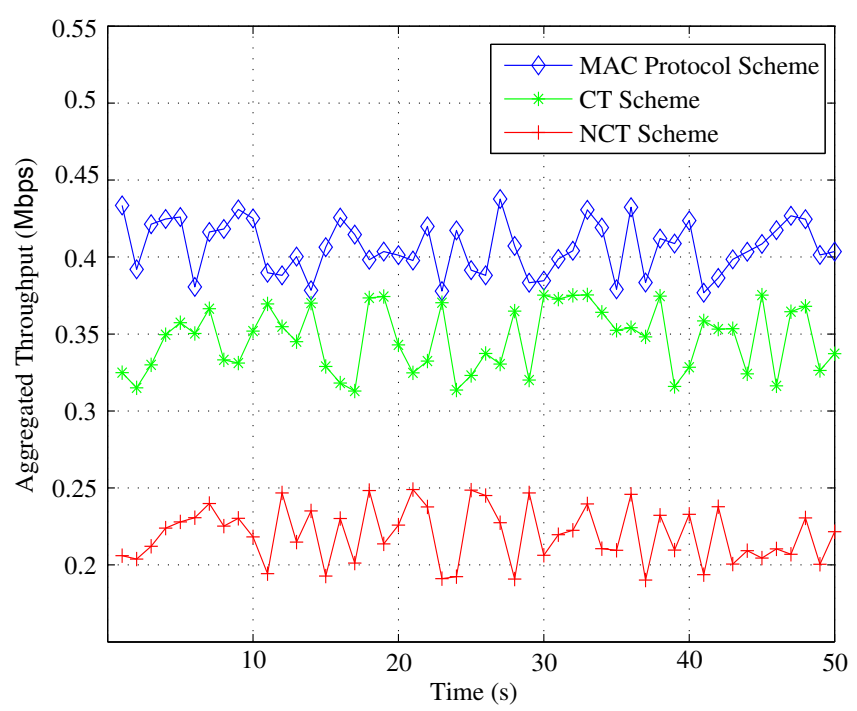

Fig. 6: Comparison of Different Schemes in Terms of Aggregated Throughput for Unoccupied Channels Equal to $2 / 3$

\section{References}

[1] S. Kim, R. Vyas, J. Bito, K. Niotaki, A. Collado, A. Georgiadis, and M. Tentzeris. Ambient RF EnergyHarvesting Technologies for Self-Sustainable Standalone Wireless Sensor Platforms. Proceedings of the IEEE, vol. 102, no. 11, pp. 1649-1666, Nov 2014.

[2] Joydev Ghosh, Sanjay Dhar Roy. Qualitative Analysis for Coverage Probability and Energy Efficiency in CognitiveFemtocell Networks Under Macrocell Infrastructure. Electronics Letters, Vol. 51, No. 17, pp.1378-1380, Aug.2015.

[3] X. Lu, P. Wang, D. Niyato, D. I. Kim, Z. Han. Wireless Networks with RF Energy Harvesting: A Contemporary Survey. IEEE Commun. Surveys Tuts. vol. 17, no. 2, Second Quarter 2015.

[4] Hoang-Sy Nguyen, Dinh-Thuan Do, Miroslav Voznak. Two-way relaying networks in green communications for 5G: Optimal throughput and tradeoff between relay distance on power splitting-based and time switchingbased relaying SWIPT. AEU - International Journal of Electronics and Communications Volume 70, Issue 12, December 2016, Pages 1637-1644

[5] Wu He, Hongjiang Lei, Gaofeng Pan. Performance modeling and analysis on conditional DF relaying scheme over Nakagami- $m$ fading channels with integral $m$. AEU International Journal of Electronics and Communications Volume 70, Issue 6, June 2016, Pages 743-749.

[6] Rui Zhang, Chin Keong Ho. MIMO Broadcasting for Simultaneous Wireless Information and Power Transfer. IEEE Transactions on Wireless Communications, Vol.12, Issue.5, pp. 1989-2001, May 2013.

[7] P. Grovez, A. Sahai. Shannon meets tesla: Wireless information and power transfer. Information Theory Proceedings (ISIT). 2010 IEEE International Symposium on, June 2010, pp. 2363-2367.

[8] Zhi Ang Eu, Winston K. G. Seah, Hwee-Pink Tan. A Study of MAC Schemes for Wireless Sensor Networks Powered by Ambient Energy Harvesting. 4th International ICST Conference on Wireless Internet.

[9] Fan Yun, Bin Liu, Lei Zou, Chang Wen Chen. Performance Analysis of IEEE 802.15.6 MAC Protocol in WBAN with Energy Harvesting Nodes. 11th International Conference on Body Area Networks

[10] Pham Ngoc Son; Hyung Yun kong. Cooperative communication with energy-harvesting relays under physical layer security. IET Communications, Volume: 9, Issue: 17, pp. 2131 - 2139, Nov 2015. 